\title{
American College of Rheumatology Pediatric Core Set for Juvenile Arthritis
}

National Cancer Institute

\section{Source}

National Cancer Institute. American College of Rheumatology Pediatric Core Set for

Juvenile Arthritis. NCI Thesaurus. Code C121341.

A set of criteria used to assess clinical response (improvement) or worsening (flare), in juvenile idiopathic arthritis clinical trials. 